\title{
Phospho-dependent recruitment of the yeast NuA4 acetyltransferase complex by MRX at DNA breaks regulates RPA dynamics during resection
}

\author{
Xue Cheng ${ }^{\mathrm{a}, 1}$, Olivier Jobin-Robitaille ${ }^{\mathrm{a}, 1}$, Pierre Billon ${ }^{\mathrm{a}, 2}$, Rémi Buisson ${ }^{\mathrm{a}, 3}$, Hengyao Niu ${ }^{\mathrm{b}, 4}$, Nicolas Lacoste $^{\mathrm{a}, 5}$, \\ Nebiyu Abshiru, ${ }^{c, d}$, Valérie Côtéa, Pierre Thibault ${ }^{c, d}$, Stephen J. Kron ${ }^{\mathrm{e}}$, Patrick Sung ${ }^{b}$, Christopher J. Brandli, \\ Jean-Yves Masson ${ }^{\mathrm{a}}$, and Jacques Côté ${ }^{\mathrm{a}, 6}$
}

\begin{abstract}
aSt. Patrick Research Group in Basic Oncology, Laval University Cancer Research Center, Centre de Recherche du Centre Hospitalier Universitaire de QuébecAxe Oncologie, Québec City, QC G1R 3S3, Canada; ${ }^{b}$ Department of Molecular Biophysics and Biochemistry, Yale University School of Medicine, New Haven, CT 06520; ' Institute for Research in Immunology and Cancer, Université de Montréal, Montréal, QC H3C 3J7, Canada; ${ }^{\circ}$ Département de Pathologie et Biologie Cellulaire, Université de Montréal, Montréal, QC H3C 3J7, Canada; 'Department of Molecular Genetics and Cell Biology, The University of Chicago, Chicago, IL 60637; and fDepartment of Biochemistry, The University of Western Ontario, London, ON N6A 5C1, Canada
\end{abstract}

Edited by Fred M. Winston, Harvard Medical School, Boston, MA, and approved August 21, 2018 (received for review April 16, 2018)

The KAT5 (Tip60/Esa1) histone acetyltransferase is part of NuA4, a large multifunctional complex highly conserved from yeast to mammals that targets lysines on $\mathrm{H} 4$ and H2A (X/Z) tails for acetylation. It is essential for cell viability, being a key regulator of gene expression, cell proliferation, and stem cell renewal and an important factor for genome stability. The NuA4 complex is directly recruited near DNA double-strand breaks (DSBs) to facilitate repair, in part through local chromatin modification and interplay with 53BP1 during the DNA damage response. While NuA4 is detected early after appearance of the lesion, its precise mechanism of recruitment remains to be defined. Here, we report a stepwise recruitment of yeast NuA4 to DSBs first by a DNA damage-induced phosphorylation-dependent interaction with the Xrs2 subunit of the Mre11-Rad50-Xrs2 (MRX) complex bound to DNA ends. This is followed by a DNA resection-dependent spreading of NuA4 on each side of the break along with the ssDNA-binding replication protein $A$ (RPA). Finally, we show that NuA4 can acetylate RPA and regulate the dynamics of its binding to DNA, hence targeting locally both histone and nonhistone proteins for lysine acetylation to coordinate repair.

chromatin | lysine acetylation | DNA double-strand break | NuA4 | RPA

$\mathbf{N}$ uA4 is a highly conserved multisubunit histone acetyltransferase (HAT) complex responsible for the acetylation of nucleosomal histone $\mathrm{H} 4$ and $\mathrm{H} 2 \mathrm{~A}$ (and variants H2A.Z/X) through its KAT5 (Esa1/Tip60) catalytic subunit (1). As the only essential HAT in yeast, NuA4 regulates through chromatin modification the expression of specific genes from transcription activation to elongation $(2,3)$, but it also regulates lifespan and autophagy through acetylation of nonhistone proteins (4-6). In higher eukaryotes, NuA4/TIP60 is essential for the maintenance and renewal of stem cells, a key regulator of cell homeostasis and response to stress, and a haplo-insufficient tumor suppressor (1). NuA4 is also critical for genome stability, as it is rapidly recruited to chromatin surrounding a DNA double-strand break (DSB) to facilitate repair (7), regulate DNA damage signaling, and counteract 53BP1 to favor homologous recombination $(\mathrm{HR})(8,9)$.

The repair of DSBs is critical for genome stability, and several studies over the past decade have implicated chromatin modifiers and remodelers in that process throughout eukaryotes. Histone phosphorylation, methylation, and ubiquitination have been shown to play key roles in the association of repair factors to the DNA damage site (9-11). Since NuA4 can interact with DNA damageinduced phosphorylated $\mathrm{H} 2 \mathrm{~A}(\mathrm{X})[\gamma \mathrm{H} 2 \mathrm{~A}(\mathrm{X})]$ around DSBs (7), we proposed that this interaction was important for $\mathrm{NuA} 4$ appearance at the break and that acetylation by NuA4 affects later steps of chromatin remodeling and repair $(12,13)$. In this study, we dissected mechanisms of NuA4 recruitment and function at DNA breaks, uncovering a stepwise process initiated by a critical phosphodependent interaction with the Mre11-Rad50-Xrs2 (MRX) complex followed by spreading during DNA resection and regulation of replication protein A (RPA) dynamics through lysine acetylation.

\section{Results}

The MRX Complex Is Required for Recruitment of NuA4 at a DNA Break. To understand the exact mechanism of NuA4 recruitment around DSBs, we performed chromatin immunoprecipitation (ChIP) experiments with an inducible DSB system (pGAL-HO, hml $\Delta / h m r \Delta$ ) in different mutant backgrounds. Eaf1 antibody was used, since it is the only subunit unique to NuA4 (14). Consistent with previous results, we observed NuA4 enrichment on both sides of the DNA break in wild-type cells after

\section{Significance}

Double-strand breaks (DSBs) in the DNA are the most dangerous type of damage and must be repaired to maintain genome integrity, avoiding disease-linked mutations and cancer initiation/ progression. The NuA4 histone acetyltransferase complex is recruited to DNA DSBs to facilitate repair through modification of the chromatin surrounding the damage, but its recruitment mechanism is poorly understood. Here, we report that the DNA damage sensor complex Mre11-Rad50-Xrs2 physically recruits NuA4 to the break sites followed by bidirectional spreading linked to the DNA end resection required for error-free repair by homologous recombination. During that process, NuA4 can acetylate ssDNA-binding replication protein A (RPA), leading to its displacement.

Author contributions: X.C., O.J.-R., P.B., R.B., V.C., P.T., S.J.K., C.J.B., J.-Y.M., and J.C. de signed research; X.C., O.J.-R., P.B., R.B., N.L., N.A., V.C., C.J.B., and J.C. performed research H.N., N.L., S.J.K., P.S., C.J.B., and J.-Y.M. contributed new reagents/analytic tools; X.C., O.J.-R., P.B., R.B., N.A., V.C., P.T., C.J.B., and J.C. analyzed data; and X.C. and J.C. wrote the paper.

The authors declare no conflict of interest.

This article is a PNAS Direct Submission.

Published under the PNAS license.

${ }^{1}$ X.C. and O.J.-R. contributed equally to this work

${ }^{2}$ Present address: Department of Genetics and Development, Herbert Irving Comprehensive Cancer Center, Columbia University Medical Center, New York, NY 10032.

${ }^{3}$ Present address: Department of Biological Chemistry, University of California, Irvine, CA 92697.

${ }^{4}$ Present address: Department of Molecular and Cellular Biochemistry, Indiana University, Bloomington, IN 47405

${ }^{5}$ Present address: Institut NeuroMyoGene, Ecole Normale Supérieure de Lyon, 69364 LYON cedex 07, France.

${ }^{6}$ To whom correspondence should be addressed. Email: Jacques.Cote@crhdq.ulaval.ca.

This article contains supporting information online at www.pnas.org/lookup/suppl/doi:10. 1073/pnas.1806513115/-/DCSupplemental.

Published online September 17, 2018. 
induction of the HO cleavage at the $M A T$ locus (Fig. $1 A$ and $S I$ Appendix, Fig. S1A). However, when we used a mutant deficient for the DNA damage-induced phosphorylation of local $\mathrm{H} 2 \mathrm{~A}$ (H2AS129A), we still detected very efficient recruitment of $\mathrm{NuA} 4$ around the DSB even in the absence of $\gamma \mathrm{H} 2 \mathrm{~A}(\mathrm{X})$, as it was previously reported for chromatin remodelers (Fig. $1 B$ and $S I$ Appendix, Fig. S1 $B$ and $C$ ) (15). These results indicate that NuA4 interaction with $\gamma \mathrm{H} 2 \mathrm{~A}$ is not responsible for $\mathrm{NuA} 4$ initial recruitment at DSBs, but more likely for its local stabilization/ accumulation. Thus, another molecular interaction is required for $\mathrm{NuA} 4$ early association with DNA breaks.

The Tra1 subunit of yeast NuA4 and its human homolog TRRAP have similar domain arrangements as the pioneer DNA damagesensing kinases Mec1 and Tel1 in yeast and ATR, ATM, and DNAPKcs in humans (Fig. 1C). These kinases are members of the phosphoinositide 3-kinase-related protein kinase (PIKK) family and function as damage signal transducers, with Tel1/ATM and DNAPKcs being important for DSB repair and with Mec1/ATR responding to ssDNA generated by lesion processing (16). Importantly, the activation of these transducer proteins requires their recruitment to the damage sites by sensor proteins. For example, while the Xrs2/Nbs1 subunit of the MRX/N complex recruits Tel1/ATM, Mec1/ATR recruitment requires Lcd1/ATRIP, and DNA-PKcs recruitment is mediated by the Ku70-Ku80 dimer (SI Appendix, Fig. S1D) $(17,18)$. Consistent with the notion of PIKK recruitment for activation, Tra1, which lacks kinase activity, has been linked to the recruitment of $\mathrm{NuA} 4$ to promoters through its interaction with the Gal4 and Gen4 transcription activators (19-21). Thus, it was tempting to speculate that the factors known to recruit PIKK proteins could be responsible for NuA4 recruitment (SI Appendix, Fig. $\mathrm{S} 1 D$ ). To test this possibility, we performed ChIP in different mutants of the candidate recruitment factors. We observed that, in the $y k u 80 \Delta$ background, there is still recruitment of NuA4 at the break (Fig. $1 D$ and $S I$ Appendix, Fig. S1E). In parallel, the deletion of $L C D 1 / A T R I P$ did not significantly affect NuA4 recruitment (Fig. $1 E$ and SI Appendix, Fig. S1E). By contrast, the deletion of XRS2 totally abolished the appearance of NuA4 around the DSB (Fig. $1 F$ and SI Appendix, Fig. S1F), which was confirmed with the two other subunits of the MRX complex, Mre11 and Rad50 (Fig. $1 G$ and $H$ and SI Appendix, Fig. S1G). These results indicate that NuA4 recruitment is specifically dependent on the MRX complex, perhaps through an interaction between MRX and Tra1.

DNA Damage Induces a Phospho-Dependent Interaction of NuA4 with Xrs2. To test whether there could be a direct interaction between Tra1 and different PIKK recruitment factors, we performed GST pull-down assays using recombinant proteins. We observed a direct interaction between Tra1 PI3K-FATC domain and the $\mathrm{N}$ terminus of Xrs2 as well as Lcd1 (Fig. $2 A$ and SI Appendix, Fig. $\mathrm{S} 2 A$ ). To verify these results in the context of the native NuA4 complex, recombinant recruitment factors were used to pull down NuA4 complex purified from yeast cells (SI Appendix, Fig. S2A).
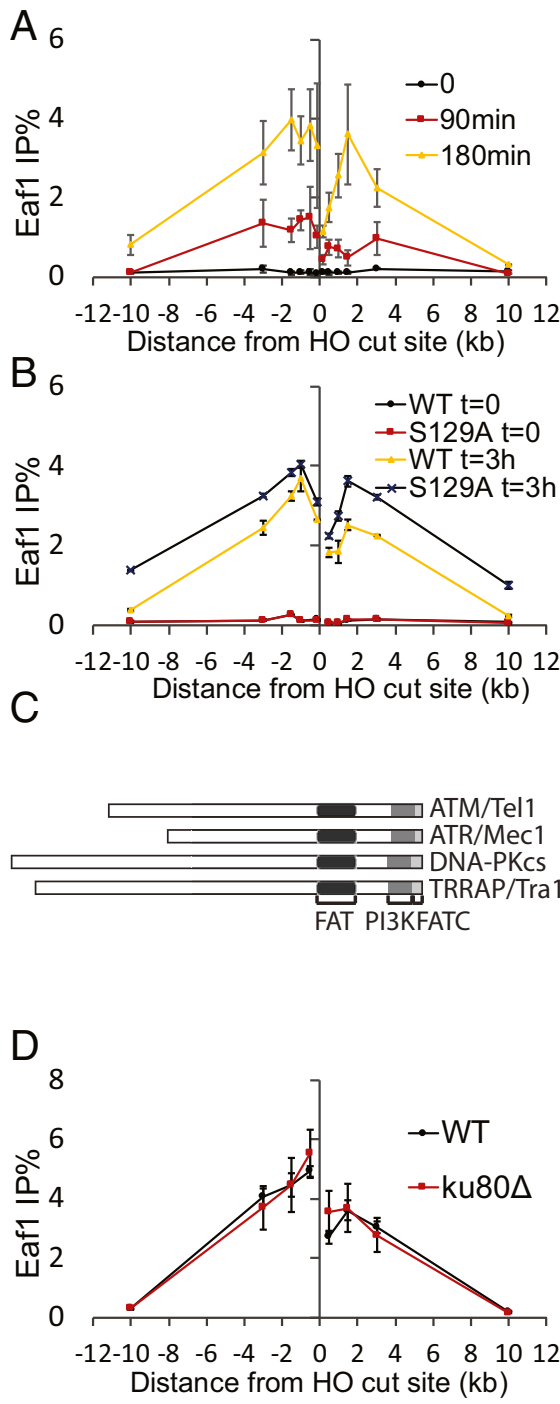

Cheng et al.
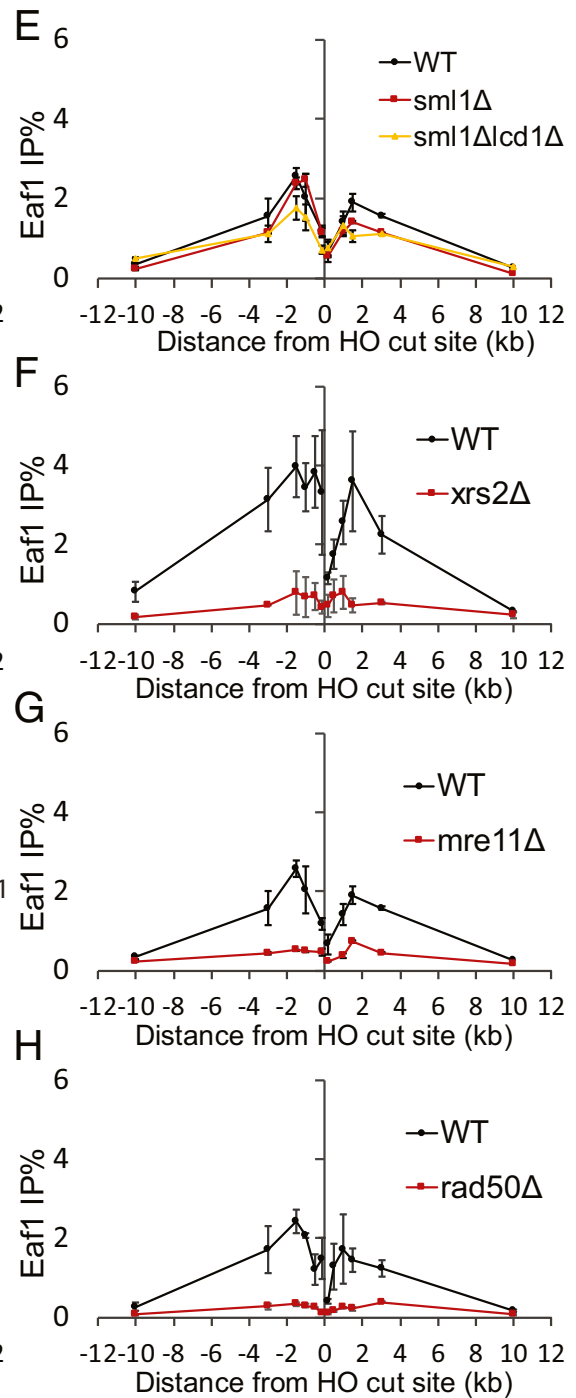

Fig. 1. NuA4 recruitment around DNA breaks depends on MRX. (A) ChIP assay of Eaf1 with time course DSB induction at MAT locus (0, 90, $180 \mathrm{~min})$. Precipitated DNA was analyzed by qPCR with primers scanning both sides of the break up to $10 \mathrm{~kb}$. (B) ChIP-qPCR assay as in $A$ in the wild type (WT) and H2AS129A mutant with no damage or on $3 \mathrm{~h}$ of $\mathrm{HO}$ induction. (C) Schematic representation of domain arrangement of TRRAP/Tra1 and PIKK family kinases ATM/Tel1, ATR/Mec1, and DNA-PKcs. $(D-H)$ ChIPqPCR assay as in $A$ in the WT and yku80s (D), $s m / 1 \Delta, s m / 1 \Delta / c d 1 \Delta(E), x r s 2 \Delta(F), \operatorname{mre} 11 \Delta(G)$, and $\operatorname{rad} 50 \Delta(H)$ strains on 3-h DSB induction. Error bars represent the range from two biological replicates $(B, E, G$, and $H)$ or $\mathrm{SE}$ from three biological replicates $(A, D$, and $F)$. 

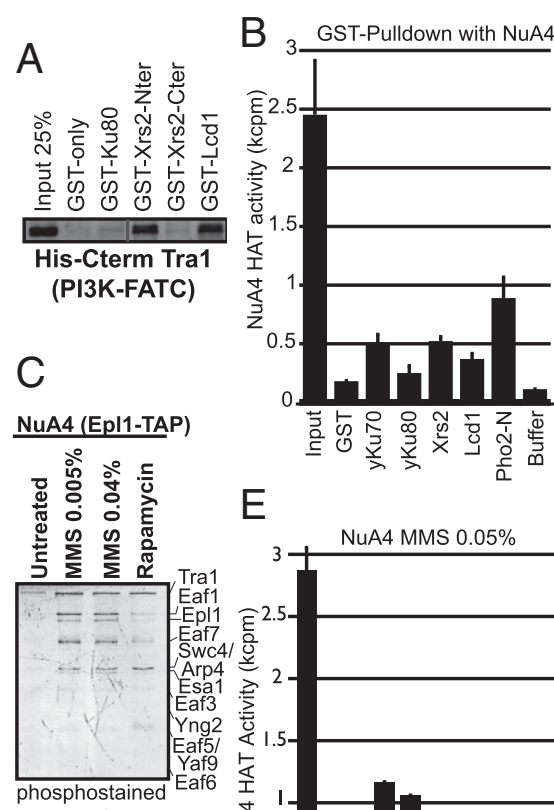

gel
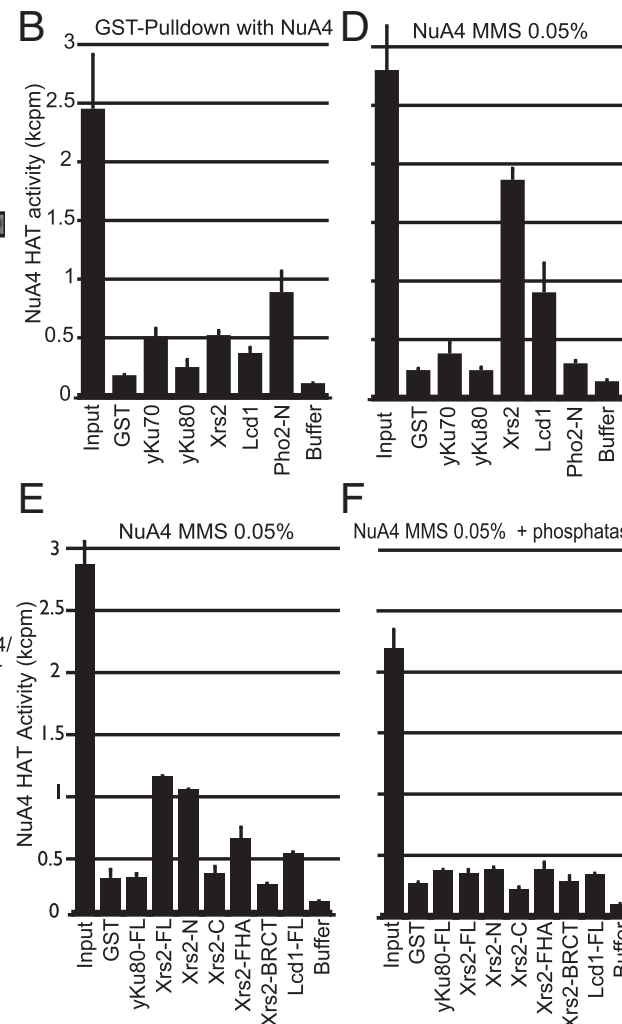

$F$
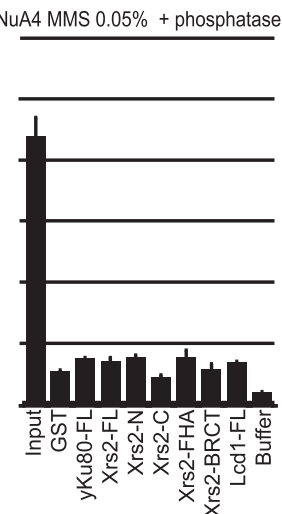

Fig. 2. DNA damage-induced phosphorylation of NuA4 is required to interact with Xrs2. (A) Pull-down assay with indicated recombinant proteins was visualized by Western blot. $(B)$ Pull down of NuA4 with recombinant GST-yKu70, yKu80, Xrs2, Lcd1, and Pho2 was measured by HAT assay. NuA4 was purified from undamaged cells. (C) Purified NuA4 from untreated MMS-treated, or rapamycin-treated cells ( $2 \mathrm{~h}$ ) was stained with Pro-Q diamond phosphoprotein gel stain (P33300; Invitrogen) following the manufacturer's instruction. ( $D-F$ ) Pull-down assay as in $A$ with NuA4 purified from MMS-treated cells. In $F, 5 \mu \mathrm{L}$ of $\lambda$-phosphatase (P0753; NEB) was added to the reaction, incubated at $30{ }^{\circ} \mathrm{C}$ for $30 \mathrm{~min}$, and repeated once. Error bars represent the range from two technical replicates.

The Pho2 transcription factor served as a positive control, since it directly interacts with NuA4 (22). As none of the damage-sensing recruitment factors pulled down $\mathrm{NuA} 4$ activity (Fig. $2 B$ ), we considered the possibility that the interaction could be mediated by DNA damage-induced phosphorylation events. Indeed, NuA4 purified from cells treated with the DNA damaging agent methylmethane sulfonate (MMS) showed a distinct phosphorylation pattern for several subunits (such as Eaf1, Epl1, Eaf7, and Esa1) compared with untreated or treated with rapamycin (Fig. 2C). This NuA4 complex modified in conditions of DNA damage response can now be efficiently pulled down by Xrs2 and to a lesser extent, by Lcd1 (Fig. 2D). Additional analysis identified the N-terminal FHA phospho-binding domain of Xrs2 as the interaction interface (Fig. $2 E$ ). Pretreating purified $\mathrm{NuA} 4$ with $\lambda$-phosphatase abolished interaction with Xrs2, confirming that it depends on NuA4 phosphorylation induced by DNA damage (Fig. $2 F$ ). We attempted to identify the DNA damage-induced phosphorylation sites on NuA4 subunits responsible for the interaction with Xrs2. Unfortunately, mutation of the Mec1/Tel1-dependent phosphorylation sites that have been mapped on NuA4 subunits, either alone or in combination, failed to render cells sensitive to DNA damaging agents (SI Appendix, Fig. S2 $B-D)(23-26)$. Nevertheless, our results support a phospho-dependent recruitment of NuA4 at DNA breaks by the Xrs2 FHA domain, potentially through the Tra1 subunit. Although the FHA domain of Xrs2/Nbs1 is important for phospho-dependent interactions with several repair factors (18) and $\mathrm{NuA} 4$, the phosphorylation sites in the NuA4 complex that are germane for DNA damage recruitment remain to be identified. Interestingly, the FHA domain of Xrs2/Nbs1 interacts with CK2 phosphorylation motifs $(27,28)$, and Tra1 contains related consensus CK2 phosphorylation sites, offering a potential avenue for future investigation.

After Initial Recruitment, NuA4 Spreads on Each Side of the Break During End Resection. The MRX complex promotes HR, which mainly takes place in the S/G2 phases of the cell cycle (29). To test whether NuA4 recruitment at DNA breaks is similarly cell cycle dependent, NuA4 enrichment was measured in cells arrested in G1 or G2/M. We observed strong NuA4 recruitment at the $\mathrm{HO}$ DSB in G2/M cells as well as in asynchronous cells, while little recruitment was detected in G1 blocked cells (Fig. $3 A$ and $S I$ Appendix, Fig. S3A). Furthermore, since NuA4 recruitment is increased in $y k u 70 \Delta$ or $y k u 80 \Delta$ cells in $\mathrm{G} 1$ (Fig. $3 B$ ), and Ku inhibits the resection of DNA ends (30), we asked whether the increase of $\mathrm{NuA} 4$ is due to elevated resection in these mutants. Indeed, inhibiting resection by overexpression of the CDK1 inhibitor Sic1 (31) greatly compromised NuA4 recruitment (Fig. 3B).

To further determine the relationship between DNA end resection and $\mathrm{NuA} 4$ recruitment, we measured $\mathrm{NuA} 4$ enrichment in cells mutated for resection factors. Individually deleting EXO1, SGS1, or $S A E 2$ did not significantly diminish recruitment (Fig. $3 C$ ). However, by deleting both $E X O 1$ and $S G S 1$, which mediate distinct resection mechanisms in parallel, we observed a loss of NuA4 recruitment at $1 \mathrm{~kb}$ from the DSB (Fig. $3 C$ and SI Appendix, Fig. S1F). This was accompanied by a clear retention of nucleosomes and strong accumulation of $\gamma \mathrm{H} 2 \mathrm{~A}$ in close proximity to the break in contrast to the usual depletion seen in wild-type conditions (SI Appendix, Fig. S3 B and $C$ ). An extended analysis of the region surrounding the DNA break revealed that $\mathrm{NuA} 4$ recruitment still occurred but became confined to the close proximity to the DSB instead of the several kilobases recruitment domain observed in wild-type cells (Fig. 3D) Together, these results indicate that $\mathrm{NuA} 4$ recruitment at DNA breaks occurs in a two-step mechanism, whereby $\mathrm{NuA} 4$ is first recruited by MRX on its DNA damage-induced phosphorylation and then spreads during DNA end resection on each side of the DSB.

The single-stranded DNA (ssDNA) structure generated during resection serves as the recruitment platform for the ssDNAbinding protein RPA, which is subsequently replaced by the
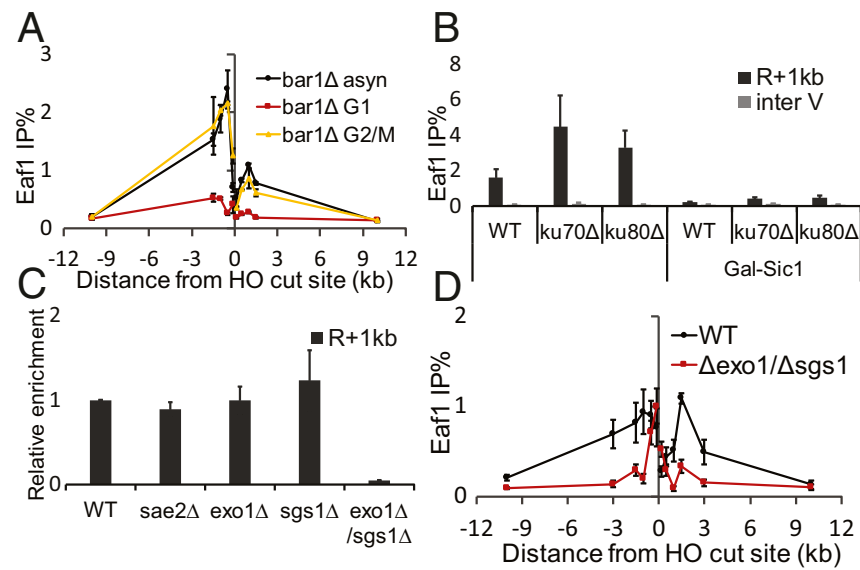

Fig. 3. NuA4 recruitment at DNA breaks occurs mainly in $\mathrm{G} 2$ and is followed by spreading during resection. $(A)$ ChIP-qPCR of Eaf1 after 3-h HO induction in bar1 $\Delta$ cells with/without synchronization. (B) ChIP-qPCR of Eaf1 in wild-

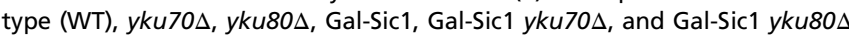
cells. Cells were synchronized in G1 with $15 \mu \mathrm{g} / \mathrm{mL} \alpha$-factor for $3 \mathrm{~h}$ followed by $3 \mathrm{~h}$ of $2 \%$ galactose induction before cross-linking. (C) ChIP-qPCR of Eaf1 in the WT and indicated resection mutants. WT value was normalized to one. (D) ChIP-qPCR of Eaf1 in the WT and exo1 $1 \Delta$ sgs $1 \Delta$ strain. Error bars represent the range from two biological replicates. 
recombinase Rad51 for the catalysis of DSB repair by HR (32). We considered the possibility that NuA4 spreading during resection might occur through direct binding to ssDNA. The NuA4 subunit Swc4 contains an SANT/Myb-like DNA-binding domain related to the one present in telomere-binding protein Tbf1 (14, 33). Using a gel shift assay with recombinant Swc4, we detected some binding to ssDNA and a DNA molecule with a $3^{\prime}$ overhang mimicking a resected DNA end (SI Appendix, Fig. S4A). Furthermore, yeast cells carrying a point mutation in the Swc4 SANT domain (swc4-23, thermosensitive) (34) are hypersensitive to DNA damage (SI Appendix, Fig. S4B) and show a significant decrease in NuA4 enrichment near the HO break (SI Appendix, Fig. S4 $C$ and $D)$. These results suggest that NuA4 may spread on ssDNA generated during DNA end resection through direct binding. Additional detailed studies will be required to verify this hypothesis.

NuA4 and RPA Interact on ssDNA During Resection. Since RPA is present on the ssDNA during resection, we next asked whether there are physical and functional interactions between RPA and $\mathrm{NuA} 4$. NuA4 coimmunoprecipitated with Rfa1, the largest subunit of RPA, from whole-cell extracts but only when cells are treated with MMS (Fig. 4A). Reciprocal immunoprecipitation (IP) confirmed this DNA damage-dependent interaction. To verify this result within the cellular setting, we used a ChIP-mass spectrometry (MS) approach to analyze proteins that colocalize with RPA after DSB induction. DNA repair factors and NuA4 components were clearly detected with RPA upon DSB induction (Fig. 4B). Interestingly, ChIP-qPCR analysis revealed increased NuA4 recruitment at the HO break in RPA mutant cells (rfal-D228Y), a mutant with less affinity for ssDNA without affecting resection per se, which we also confirmed by ChIP assay (Fig. $4 C$ and SI $A p$ pendix, Fig. S4 $E$ and $F)(35,36)$. These results suggest that, while RPA is not required for NuA4 spreading during resection, there may be a functional incompatibility between NuA4 and RPA on the ssDNA. Importantly, repair factors Rad51 and Rad52, required for HR (both) or single-strand annealing (Rad52), did not significantly affect $\mathrm{NuA} 4$ binding around the $\mathrm{HO}$ break (Fig. $4 D$ and $E$ ),

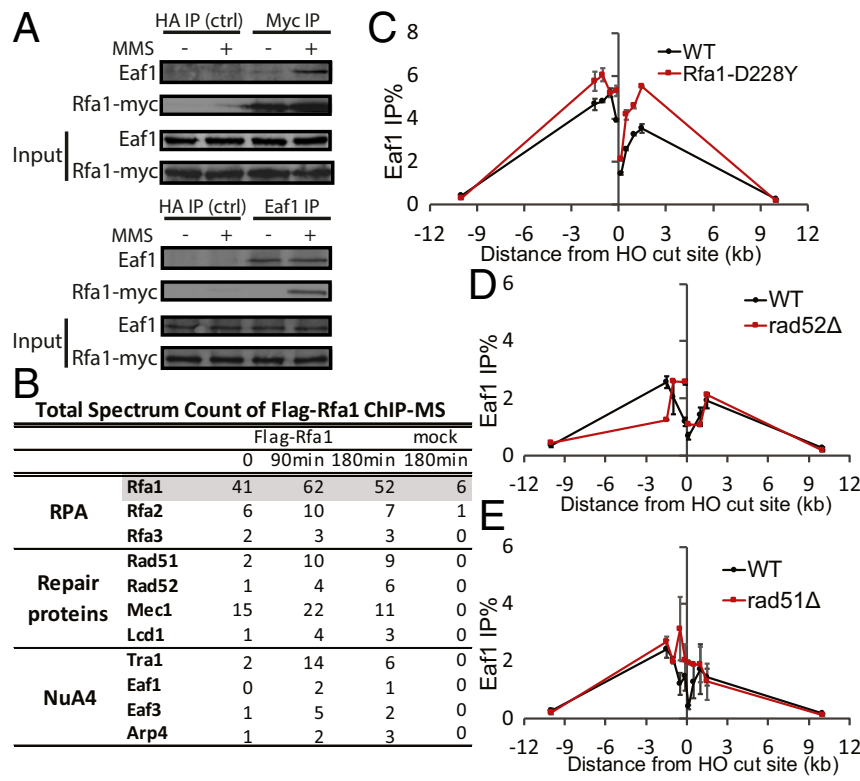

Fig. 4. NuA4 interacts with RPA on DNA damage. (A) Co-IP and reciprocal co-IP of 13myc-Rfa1 and Eaf1 from whole-cell extracts of cells untreated or treated with MMS $0.05 \%$ for $1 \mathrm{~h}$. HA IP serves as the negative control. (B) List of proteins and number of total peptides detected in ChIP of Flag-Rfa1 followed by MS analysis from cells after 0,90 , or $180 \mathrm{~min}$ of $\mathrm{HO}$ induction (C-E) ChIP-qPCR of Eaf1 after 3-h HO induction in the wild type (WT) and rfa1-D228Y $(C), \operatorname{rad52} \Delta(D)$, and $\operatorname{rad51\Delta }(E)$. Error bars represent the range from two biological replicates. nor did the 9-1-1 clamp or clamp loader (SI Appendix, Fig. S4 $G$ and $H$ ). Future studies will determine if NuA4 and ATP-dependent chromatin remodelers spread on resected DNA ends via a similar mechanism (15).

NuA4 Acetylates RPA and Modulates Its Binding to ssDNA. To further investigate the functional cross-talk between RPA and NuA4 during DNA end resection, we used a reconstituted in vitro system with purified NuA4 and RPA complexes (SI Appendix, Fig. S5A) and ssDNA bound to magnetic beads. RPA was first preloaded on the DNA, and NuA4 was then added to measure possible competition between the two complexes for the ssDNA (Fig. 5A). Just adding NuA4 complex to RPA-coated ssDNA did not affect the RPA (Rfa2) signal associated with the beads (Fig. $5 B$ and $C$, lanes 1-3). Strikingly, the addition of AcCoA led to a clear NuA4dependent displacement of RPA from the ssDNA (Fig. $5 B$ and $C$, lanes 4-6). The extent of the effect correlates with the incubation time (Fig. $5 B$ ) and the amount of NuA4 in the reaction (Fig. $5 C$ ), but the displacement was not observed in Esa1 catalytically dead mutants (SI Appendix, Fig. S5B) $(37,38)$. These results with a purified reconstituted system imply that NuA4 targets RPA for acetylation, which affects its interaction with ssDNA. To confirm that NuA4-dependent RPA acetylation occurs in vivo, we performed acetyllysine IP in extracts from wild-type and thermosensitive esa1 mutant cells [esa1-L254P (39)] at nonpermissive temperature (Fig. $5 D$ and SI Appendix, Fig. S5C). Less RPA signal was detected in the esa1 mutant sample in the presence of DNA damage, arguing that NuA4 is indeed involved in RPA acetylation in vivo. Different studies, including our work, have identified multiple in vivo lysine acetylation sites in the heterotrimeric yeast and human RPA (SI Appendix, Fig. S5 $D-F$ ). To determine whether acetylation of RPA is functionally relevant, we generated mutants within residues that are acetylated in yeast or in human RPA on lysines that are conserved in yeast (Rfa1 K259, K463, K494) (40-42) (our work in SI Appendix, Fig. S5 $D-F$ ). Mutation of lysine to arginine blocks acetylation but conserves the positive charge, while glutamine residues mimic acetylated lysines. Substitution of all three aforementioned lysines with arginine did not affect cell growth or the cellular resistance to DNA damage (Fig. 5E and SI Appendix, Fig. S5 $G$ and $H$ ). Similarly, substituting them for three glutamine residues does not affect normal cell growth or resistance to UV, indicating that RPA function on ssDNA during replication fork progression is not affected, and also, its role in nucleotide excision repair is not affected (Fig. 5E, Top Left and SI Appendix, Fig. $\mathrm{S} 5 H$ ). In contrast, growth on media containing chemicals that cause DNA breaks is as strongly impaired as seen for sae $2 \Delta$ mutant cells (Fig. 5E). The most striking sensitivity was detected in the presence of the toposiomerase I inhibitor camptothecin (CPT), which induces DSBs arising from the collapse of DNA replication forks (Fig. 5E, Bottom Left). We then analyzed the binding of these mutant RPA molecules during resection at the HO DSB in vivo and found a clear defect of Rfa1-QQQ association near the break, in agreement with the in vitro data (Fig. $5 F$ and SI Appendix, Fig. S5I). Surprisingly, the Rfa1-RRR mutant was also affected but to a lesser extent, suggesting that dynamic acetylation/deacetylation is important for RPA function during resection. Altogether, our in vitro and in vivo data point to an important functional interaction between NuA4 and RPA, providing yet another regulatory mechanism of DNA damage response through the direct action of a chromatin-modifying enzyme on substrates beyond histones/chromatin.

\section{Discussion}

Collectively, these data lead us to propose a stepwise recruitment model of NuA4 around a DNA break (Fig. $5 G$ ). On DSB formation, the MRX sensor complex recognizes the break followed by the loading of Tel1, which transduces the DNA damage signal through its kinase activity on targets, such as histone H2A. In parallel, MRX recruits $\mathrm{NuA} 4$ that has undergone DNA damage-induced phosphorylation. The previously reported interaction of $\gamma \mathrm{H} 2 \mathrm{~A}$ with NuA4 favors its retention/accumulation. This leads to chromatin 

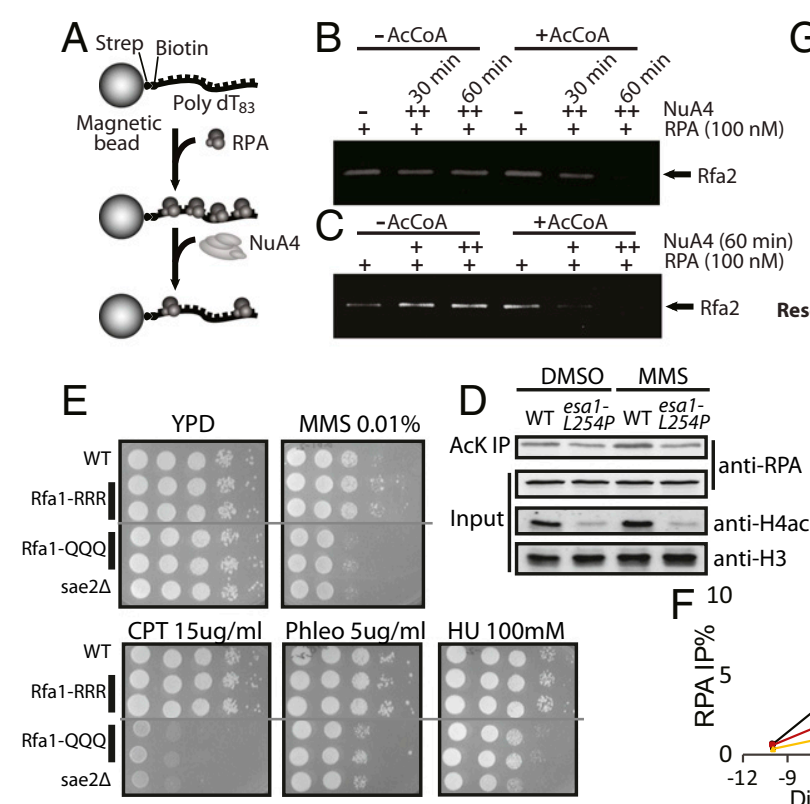

G
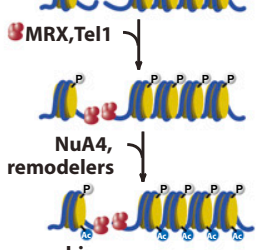

$\underset{R P A}{\operatorname{RP}}$ )

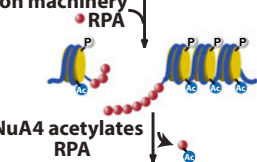

-Rad51 loading and recombination -checkpoint activation (LCd1/Mec1) -short ssDNA streches for MMEJ RPA ChIP

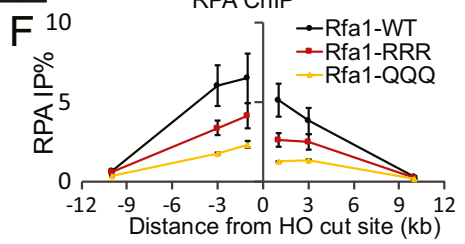

Fig. 5. NuA4 displaces RPA from ssDNA in an AcCoAdependent manner. $(A)$ Schematic representation of biotin-ssDNA pull-down assay. ( $B$ and $C$ ) Biotin-ssDNA pull-down assay under the indicated conditions was loaded onto $8 \%$ SDS/PAGE, stained by Sypro Ruby, and visualized under UV. $B$ shows different incubation times (0/30/60 $\mathrm{min})$, and $C$ shows different amounts of NuA4 added to the reaction. $(D)$ Western blot analysis with indicated antibodies of IPs with acetyllysine antibody (ICP0380; Immunechem) in whole-cell extracts (input) from wild-type (WT) and esa 1-L254P cells treated with $0.05 \%$ DMSO or MMS at $37{ }^{\circ} \mathrm{C}$ for $1 \mathrm{~h}$. $(E)$ Tenfold serial dilutions of the WT, genome-integrated Rfa1 mutants (K259/463/494), and sae2 $\Delta$ were spotted onto yeast extract peptone dextrose (YPD) or YPD containing MMS, CPT, phleomycin, or hydroxyurea (HU) at the indicated concentrations and grown at $30{ }^{\circ} \mathrm{C}$. ( F) ChIP-qPCR of RPA after 3-h HO induction in the WT and RRR/QQQ mutants. $(G)$ Model for stepwise recruitment of NuA4 around DNA breaks. acetylation around the break site to facilitate chromatin relaxation by ATP-dependent remodelers, like SWI/SNF (13) and Rvb1/2containing complexes (7). Subsequently, NuA4 spreads on each side of the DSB concomitant with DNA end resection, where it regulates the dynamics of RPA binding to ssDNA via its acetylation. Future work will address the functional consequences of RPA regulation by NuA4 during the repair of DSBs. It is possible that $\mathrm{NuA} 4$ acetylates RPA to facilitate subsequent loading of Rad51 for the mediation of HR or Lcd1-Mec1 for DNA damage checkpoint regulation. Moreover, as RPA can antagonize microhomology-mediated end joining (MMEJ) (36), RPA displacement/destabilization by NuA4 may expose only short stretches of ssDNA that can channel the DSB repair pathway away from HR and more toward MMEJ. Based on recent analysis of RPA mutants with less affinity for ssDNA, another possibility is that NuA4-dependent acetylation may favor single-strand annealing and/or regulates break-induced replication (43). All of these different possible pathways will need to be analyzed in the context of RPA acetylation.

The understanding of how a DNA damage site is repaired by the cell has been a focus of intense research because of its link to genome stability and human disease. The discovery of the initial recruitment of a chromatin modifier complex by MRX to a DNA break is an important mechanistic insight. In gene regulation, NuA4-dependent acetylation is known to promote the association and action of ATPdependent remodelers SWI/SNF, RSC, and SWR1 (1), and they are also implicated in DSB repair throughout eukaryotes $(9,44)$. Resection-dependent recruitment of NuA4 seems similar to what has been reported for a host of chromatin remodeling enzymes (15), while NuA4 activity has also been shown to regulate their recruitment $(7,13)$. Subsequent action of $\mathrm{NuA} 4$ during DNA end resection led us to uncover a regulatory step by targeting a nonhistone substrate at the heart of the DNA repair process. Based on the high degree of evolutionary conservation of these chromatin-modifying/remodeling complexes, repair proteins, and lysine acetylation sites, these mechanistic findings are expected to be conserved in higher eukaryotes.

\section{Materials and Methods}

SI Appendix, SI Materials and Methods provides details about ChIP-qPCR assay, GST pull down, gel shift assay, acetyllysine IP, and mutant Esa1/NuA4.

Yeast Strains, Materials, and MS. Yeast strains used in this study are listed in SI Appendix, Table S1 and were constructed based on standard PCR-based transformation protocol. NuA4 and RPA complexes were purified from yeast cells as previously described $(2,45)$. Analysis of phosphorylation sites on
NuA4 purified from cell treated with MMS was performed after separation on SDS/PAGE followed by in-gel digestion of protein bands. Reduction and alkylation of cysteine residues were performed with $100 \mu \mathrm{L}$ of $20 \mathrm{mM}$ tris(2 carboxyethyl)phosphine (TCEP) and $40 \mathrm{mM}$ lodoacetamide, respectively. After digestion with $2 \mu \mathrm{g}$ of trypsin in $50 \mathrm{mM}$ ammonium bicarbonate, peptides were extracted with $70 \%$ acetonitrile, dried, resuspended in $0.2 \%$ formic acid and analyzed on LTQ-Orbitrap XL MS coupled to an Eksigent nano-LC system. Raw liquid chromatography-MS/MS data were searched against a custom database with Mascot search engine v2.10 (Matrix science).

ChIP and ChIP-MS. ChIP assay was essentially performed as described previously $(2,46)$ using anti-Eaf1 (14), anti-H3 (Ab1791; Abcam), anti-RFA (AS07 214 Agrisera), and anti- $\gamma \mathrm{H} 2 \mathrm{~A}$ antibodies (07-745; Upstate). IP percentage is presented as the value of the IP sample over input. A list of primers used in qPCR is presented in SI Appendix, Table S2. SI Appendix provides a more detailed ChIP protocol. For ChIP-MS, endogenous Rfa1 was tagged with Flag and subjected to ChIP modified as follows. One milligram of chromatin was incubated with $6 \mu \mathrm{g}$ of anti-Flag M2 antibody (F1804; Sigma) overnight at $4{ }^{\circ} \mathrm{C} ; 200 \mu \mathrm{L}$ of dynabeads coated with protein G (10004D; Life Technologies) were added and placed on a rotating wheel at $4{ }^{\circ} \mathrm{C}$ for $4 \mathrm{~h}$. After washing five times with formaldehyde (FA) lysis buffer, triple Flag peptide (F4799; Sigma) was added to a final concentration of $0.5 \mathrm{mg} / \mathrm{mL}$ to elute the bound fraction from the beads. Eluted fraction was loaded to $12 \% \mathrm{Bis}$ Tris precast polyacrylamide gel, Sypro Ruby (Bio-Rad) stained, and sent for MS analysis at the Quebec Genome Center.

GST Pull-Down Assay. GST pull-down assay was performed as described previously (46). GST expression plasmids were based on pGEX-4T3 backbone and constructed by conventional cloning containing full-length proteins or partial proteins: Xrs2-Nterm (amino acids 1-423), Xrs2-Cterm (amino acids 424-854), Xrs2-FHA (amino acids 1-117), Xrs2-BRCT (amino acids 116-423), and Swc4-N-term (amino acids 1-285); 6xHis-tag expression plasmid was based on pET15b backbone and constructed by conventional cloning containing Tra1-Cterm (amino acids 3201-3744). SI Appendix provides a more detailed GST pull-down protocol.

Biotin-ssDNA Pull-Down and Gel Shift Assays. Biotin-ssDNA pull-down assay and gel shift assay with different ${ }^{32} \mathrm{P}$-labeled DNA molecules were performed essentially as previously described (47). For biotin-ssDNA pull-down assay, magnetic beads containing 5'-biotinylated ssDNA poly dT 83-mer (corresponding to the final concentration of $1 \mu \mathrm{M}$ nucleotide) were resuspended in $16 \mu \mathrm{L}$ of reaction buffer (50 mM Tris. $\mathrm{HCl}, \mathrm{pH} 7.5,1 \mathrm{mM}$ DTT, $1 \mathrm{mM}$ PMSF, $10 \mathrm{mM}$ Na-Butyrate, $0.5 \mathrm{mM} \mathrm{MgCl}, 100 \mu \mathrm{g} / \mathrm{mL}$ BSA with or without $0.15 \mathrm{mM}$

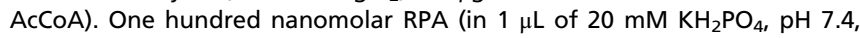
$200 \mathrm{mM} \mathrm{KCl}, 0.5 \mathrm{mM}$ EDTA, $1 \mathrm{mM} \mathrm{DTT,} \mathrm{10 \%} \mathrm{glycerol)} \mathrm{was} \mathrm{added,} \mathrm{and} \mathrm{the} \mathrm{re-}$ action was incubated for $5 \mathrm{~min}$ at $30^{\circ} \mathrm{C}$. Then, NuA4 complex (in $3 \mu \mathrm{L}$ of $20 \mathrm{mM}$ Hepes, pH 7.5, 5\% glycerol, $10 \mu \mathrm{M} \mathrm{ZnCl}_{2}, 1$ mM DTT, 1 mM PMSF, $100 \mathrm{mM} \mathrm{KCl}$ ) 
was added for 30 or $60 \mathrm{~min}$ at $30^{\circ} \mathrm{C}$. The beads were captured and washed twice with $20 \mu \mathrm{L}$ of washing buffer (50 mM Tris. $\mathrm{HCl}, \mathrm{pH}$ 7.5, $1 \mathrm{mM} \mathrm{DTT,} 1 \mathrm{mM}$ PMSF, $10 \mathrm{mM}$ Na-Butyrate, $0.5 \mathrm{mM} \mathrm{MgCl} 2,40 \mathrm{mM} \mathrm{KCl}$ ). Finally, $15 \mu \mathrm{L}$ of Laemmli buffer $1 \times$ was added followed by heating $5 \mathrm{~min}$ at $95^{\circ} \mathrm{C}$. The beads were spun down, and the supernatants were analyzed by SDS/PAGE ( $8 \%)$. The gel was stained with Sypro Ruby (Invitrogen) and visualized under UV.

NuA4-Rfa1 Co-IP. Cells were treated with $0.05 \%$ MMS (or DMSO in control) fo $1 \mathrm{~h}$. Cells were lysed in $10 \mathrm{mM}$ Tris, pH 8.0, $150 \mathrm{mM} \mathrm{NaCl}, 10 \%$ glycerol, $0.1 \%$ Nonidet P-40, $2 \mu \mathrm{g} / \mathrm{mL}$ leupeptin, $2 \mu \mathrm{g} / \mathrm{mL}$ pepstatin A, $5 \mu \mathrm{g} / \mathrm{mL}$ aprotinin, $1 \mathrm{mM}$ PMSF, $10 \mathrm{mM}$-glycerophosphate, $1 \mathrm{mM}$ Na-Butyrate, $0.5 \mathrm{mM} \mathrm{NaF}$, and $1 \mathrm{mM}$ DTT. Soluble fraction was collected by centrifugation, and each IP was carried out using $1 \mathrm{mg}$ of protein extract in addition to the antibody (anti-HA negative control, anti-myc, or anti-Eaf1 as indicated) overnight at $4{ }^{\circ} \mathrm{C}$. Beads coated with protein $\mathrm{G}$ were added to the mixture for 4

1. Steunou A-L, Rossetto D, Côté J (2014) Regulating chromatin by histone acetylation Fundamentals of Chromatin, eds Workman JL, Abmayr SM (Springer, New York), pp $147-212$.

2. Rossetto $D$, et al. (2014) Eaf5/7/3 form a functionally independent NuA4 submodule linked to RNA polymerase II-coupled nucleosome recycling. EMBO J 33:1397-1415.

3. Steunou AL, et al. (2016) Combined action of histone reader modules regulates NuA4 local acetyltransferase function but not its recruitment on the genome. $\mathrm{Mol} \mathrm{Ce} / \mathrm{BiO}$ 36:2768-2781.

4. Yi C, et al. (2012) Function and molecular mechanism of acetylation in autophagy regulation. Science 336:474-477.

5. Lin YY, et al. (2009) Protein acetylation microarray reveals that NuA4 controls key metabolic target regulating gluconeogenesis. Cell 136:1073-1084.

6. Lu JY, et al. (2011) Acetylation of yeast AMPK controls intrinsic aging independently of caloric restriction. Cell 146:969-979.

7. Downs JA, et al. (2004) Binding of chromatin-modifying activities to phosphorylated histone H2A at DNA damage sites. Mol Cell 16:979-990.

8. Jacquet $K$, et al. (2016) The TIP60 complex regulates bivalent chromatin recognition by $53 \mathrm{BP} 1$ through direct H4K20me binding and H2AK15 acetylation. Mol Cell 62 409-421.

9. Smeenk G, van Attikum H (2013) The chromatin response to DNA breaks: Leaving a mark on genome integrity. Annu Rev Biochem 82:55-80.

10. Dantuma NP, van Attikum H (2016) Spatiotemporal regulation of posttranslational modifications in the DNA damage response. EMBO J 35:6-23.

11. Wilson MD, Durocher D (2017) Reading chromatin signatures after DNA doublestrand breaks. Philos Trans R Soc Lond B Biol Sci 372:20160280.

12. Downs JA, Côté J (2005) Dynamics of chromatin during the repair of DNA doublestrand breaks. Cell Cycle 4:1373-1376.

13. Bennett G, Peterson CL (2015) SWI/SNF recruitment to a DNA double-strand break by the NuA4 and Gen5 histone acetyltransferases. DNA Repair 30:38-45.

14. Auger A, et al. (2008) Eaf1 is the platform for NuA4 molecular assembly that evolutionarily links chromatin acetylation to ATP-dependent exchange of histone $\mathrm{H} 2 \mathrm{~A}$ variants. Mol Cell Biol 28:2257-2270.

15. Bennett G, Papamichos-Chronakis M, Peterson CL (2013) DNA repair choice defines a common pathway for recruitment of chromatin regulators. Nat Commun 4:2084.

16. Lempiäinen $\mathrm{H}$, Halazonetis TD (2009) Emerging common themes in regulation of PIKKs and PI3Ks. EMBO J 28:3067-3073.

17. Falck J, Coates J, Jackson SP (2005) Conserved modes of recruitment of ATM, ATR and DNA-PKcs to sites of DNA damage. Nature 434:605-611.

18. Symington LS (2016) Mechanism and regulation of DNA end resection in eukaryotes Crit Rev Biochem Mol Biol 51:195-212.

19. Brown CE, et al. (2001) Recruitment of HAT complexes by direct activator interactions with the ATM-related Tra1 subunit. Science 292:2333-2337.

20. Knutson BA, Hahn S (2011) Domains of Tra1 important for activator recruitment and transcription coactivator functions of SAGA and NuA4 complexes. Mol Cell Biol 31 : 818-831.

21. Lin L, Chamberlain L, Zhu LJ, Green MR (2012) Analysis of Gal4-directed transcription activation using Tra1 mutants selectively defective for interaction with Gal4. Proc Nat Acad Sci USA 109:1997-2002.

22. Nourani A, Utley RT, Allard S, Côté J (2004) Recruitment of the NuA4 complex poises the $\mathrm{PHO} 5$ promoter for chromatin remodeling and activation. EMBO $\mathrm{f} 23 \cdot 2597-2607$.

23. Breitkreutz A, et al. (2010) A global protein kinase and phosphatase interaction network in yeast. Science 328:1043-1046.

24. Albuquerque $\mathrm{CP}$, et al. (2008) A multidimensional chromatography technology for in depth phosphoproteome analysis. Mol Cell Proteomics 7:1389-1396. additional hours. After extensive washes in $10 \mathrm{mM}$ Tris. $\mathrm{HCl}, \mathrm{pH} 8.0,150 \mathrm{mM}$ $\mathrm{NaCl}, 10 \%$ glycerol, $0.1 \%$ Nonidet $\mathrm{P}-40$, and $1 \mathrm{mM}$ PMSF, beads were resuspended in Laemmli buffer $1 \times$ buffer, boiled, and analyzed by SDS/PAGE followed by Western blotting using the indicated antibodies.

ACKNOWLEDGMENTS. We thank Joannie Roberge and Yan Coulombe for technical assistance. We also thank Alain Verreault and Craig Peterson for stimulating discussions and sharing reagents and Jef Boeke and Susan Gasser for sharing yeast strains. This work was supported by NIH Grants R00 ES021441 (to H.N.) and R01 GM60443 (to S.J.K. and J.C.) and Canadian Institutes of Health Research (CIHR) Grants MOP-14308 (to J.C.) and FDN143314 (to J.C.). X.C., O.J.-R., and R.B. were supported by graduate scholarships from CIHR/Banting and Best, Desjardins/Luc Bélanger/Fonds de recherche du Québec-Santé (FRQS), and Fonds de recherche du Québec-Nature et technologies (FRQNT), respectively. J.C. holds the Canada Research Chair in Chromatin Biology and Molecular Epigenetics.

25. Smolka MB, Albuquerque $\mathrm{CP}$, Chen $\mathrm{SH}$, Zhou $\mathrm{H}$ (2007) Proteome-wide identification of in vivo targets of DNA damage checkpoint kinases. Proc Natl Acad Sci USA 104: 10364-10369.

26. Bastos de Oliveira FM, et al. (2015) Phosphoproteomics reveals distinct modes of Mec1/ATR signaling during DNA replication. Mol Cell 57:1124-1132.

27. Lloyd J, et al. (2009) A supramodular FHA/BRCT-repeat architecture mediates Nbs1 adaptor function in response to DNA damage. Cell 139:100-111.

28. Williams RS, et al. (2009) Nbs1 flexibly tethers Ctp1 and Mre11-Rad50 to coordinate DNA double-strand break processing and repair. Cell 139:87-99.

29. Ceccaldi R, Rondinelli B, D'Andrea AD (2016) Repair pathway choices and consequences at the double-strand break. Trends Cell Biol 26:52-64.

30. Zhang Y, et al. (2007) Role of Dnl4-Lif1 in nonhomologous end-joining repair complex assembly and suppression of homologous recombination. Nat Struct Mol Biol 14: 639-646.

31. Ira G, et al. (2004) DNA end resection, homologous recombination and DNA damage checkpoint activation require CDK1. Nature 431:1011-1017.

32. Dubrana K, van Attikum H, Hediger F, Gasser SM (2007) The processing of doublestrand breaks and binding of single-strand-binding proteins RPA and Rad51 modulate the formation of ATR-kinase foci in yeast. J Cell Sci 120:4209-4220.

33. Aasland R, Stewart AF, Gibson T (1996) The SANT domain: A putative DNA-binding domain in the SWI-SNF and ADA complexes, the transcriptional co-repressor N-CoR and TFIIIB. Trends Biochem Sci 21:87-88.

34. Lin YY, et al. (2008) A comprehensive synthetic genetic interaction network governing yeast histone acetylation and deacetylation. Genes Dev 22:2062-2074.

35. Smith J, Rothstein R (1995) A mutation in the gene encoding the Saccharomyces cerevisiae single-stranded DNA-binding protein Rfa1 stimulates a RAD52independent pathway for direct-repeat recombination. Mol Cell Biol 15:1632-1641.

36. Deng SK, Gibb B, de Almeida MJ, Greene EC, Symington LS (2014) RPA antagonizes microhomology-mediated repair of DNA double-strand breaks. Nat Struct Mol Biol 21:405-412.

37. Allard S, et al. (1999) NuA4, an essential transcription adaptor/histone $\mathrm{H} 4$ acetyltransferase complex containing Esa1p and the ATM-related cofactor Tra1p. EMBO J 18:5108-5119.

38. Yuan $\mathrm{H}$, et al. (2012) MYST protein acetyltransferase activity requires active site lysine autoacetylation. $E M B O J$ 31:58-70

39. Clarke AS, Lowell JE, Jacobson SJ, Pillus L (1999) Esa1p is an essential histone acetyltransferase required for cell cycle progression. Mol Cell Biol 19:2515-2526.

40. Henriksen P, et al. (2012) Proteome-wide analysis of lysine acetylation suggests its broad regulatory scope in Saccharomyces cerevisiae. Mol Cell Proteomics 11: 1510-1522.

41. Mertins $P$, et al. (2013) Integrated proteomic analysis of post-translational modifications by serial enrichment. Nat Methods 10:634-637.

42. Choudhary C, et al. (2009) Lysine acetylation targets protein complexes and coregulates major cellular functions. Science 325:834-840.

43. Ruff P, Donnianni RA, Glancy E, Oh J, Symington LS (2016) RPA stabilization of single-stranded DNA is critical for break-induced replication. Cell Rep 17:3359-3368.

44. Jeggo PA, Downs JA, Gasser SM (2017) Chromatin modifiers and remodellers in DNA repair and signalling. Philos Trans $R$ Soc Lond B Biol Sci 372:20160279.

45. Niu H, et al. (2010) Mechanism of the ATP-dependent DNA end-resection machinery from Saccharomyces cerevisiae. Nature 467:108-111.

46. Cheng X, et al. (2015) Eaf1 links the NuA4 histone acetyltransferase complex to Htz1 incorporation and regulation of purine biosynthesis. Eukaryot Cell 14:535-544.

47. Buisson R, et al. (2010) Cooperation of breast cancer proteins PALB2 and piccolo BRCA2 in stimulating homologous recombination. Nat Struct Mol Biol 17:1247-1254. 